\title{
SEM \& STEM Multi-scale Characterization of Fatigue Damage in CrCoNi Medium-entropy Alloy with Fully Recrystallized Microstructure
}

Veronika Mazánová $^{1}$, Milan Heczko ${ }^{1}$, Connor Slone ${ }^{1}$, Ivo Kuběna ${ }^{2}$, Jiří Tobiáś ${ }^{3}$, Easo George ${ }^{4}$, Tomáš Kruml $^{3}$, Jaroslav Polák ${ }^{3}$ and Michael Mills ${ }^{1}$

${ }^{1}$ The Ohio State University, Columbus, Ohio, United States, ${ }^{2}$ Institute of Physics of Materials Czech Academy of Sciences, Brno, Jihomoravsky kraj, Czech Republic, ${ }^{3}$ Institute of Physics of Materials, Czech Academy of Sciences, Brno, Jihomoravsky kraj, Czech Republic, ${ }^{4}$ Oak Ridge National Laboratory, Oak Ridge, Tennessee, United States

High-entropy alloys (HEA)/multi-principal element alloys (MPEA) became a topic of very high interest among research groups all over the world. However, despite the effort which has been ongoing for several years, understanding and application of these new materials in real service conditions is presently lacking. Research in the field of MPEAs has been devoted to investigation of monotonic mechanical properties. Nevertheless, components in real service are usually exposed to cyclic external forces and loads, and thus understanding of fatigue damage and behavior is of significant interest.

The fatigue damage of material is closely related to the initial microstructural state and its evolution. Within the process, localized bands of cyclic plastic deformation with a specific substructure, so-called persistent slip bands (PSBs) arise and lead to the development of a characteristic surface relief in the form of persistent slip markings (PSMs), and subsequently to the initiation of fatigue cracks [1]. In order to be able to predict cyclic stress-strain response and the fatigue life of the components at macroscopic level, it is essential to understand the physically-based microstructure-related mechanisms acting both in the bulk and on the surface of the material.

A sophisticated experimental workflow has been designed to investigate the effects of tunable fully (RX) and partially recrystallized (PRX) heterostructures on the fatigue performance of CrCoNi alloy (see Fig. 1) [2]. Cold-rolling and annealing steps were combined to produce different initial microstructural states of the alloy [3]. While RX state consists of recrystallized equiaxed grains of $24 \mu \mathrm{m}$ in average size, PRX heterostructure presents composite of large deformation twins rich non-RX grains and fine fully recrystallized grains of $1.6 \mu \mathrm{m}$ in diameter. Special small cylindrical specimens with electropolished surface were prepared and tested in symmetric fully reversed strain-controlled cyclic loading at room temperature. To preserve all necessary information contained on the surface the whole tested specimens were put into the large chamber of dual-beam scanning electron microscope (SEM) immediately after mechanical testing. The developed surface relief was investigated. Combination of electron backscatter diffraction (EBSD), electron channeling contrast imaging (ECCI) and focused ion beam (FIB) was used to identify the sites of the interest. The profiles of PSMs developed on the surface were observed and documented simultaneously with the underlying microstructure using the technique of site- and orientation-specific TEM surface lamella preparation by FIB. Thin foils were observed in scanning transmission electron microscope using (STEM) diffraction contrast (DCI). Burgers vector, character and orientation of defects were determined.

The real shape and the profile characteristics of the surface relief were reconstructed in "3D" using described experimental framework based on mutual collaboration of several state-of-the-art electron microscopy techniques. Obtained experimental results and observations were compared and discussed with findings collected in case of other materials and simple metals as well as theoretical models of fatigue crack initiation [1,2]. The role of annealing and deformation twin boundaries within the failure 
mechanisms of $\mathrm{CrCoNi}$ was explained. Key aspects leading to the cyclic plastic slip localization followed up by the fatigue crack initiation and the final failure of $\mathrm{CrCoNi}$ medium-entropy alloy in both $\mathrm{RX}$ and PRX conditions were identified. This is a starting point for development/design of advanced alloys with ultra-high monotonic and cyclic strength while preserving a good ductility, resistance to cyclic plastic deformation and overall fatigue life [4].

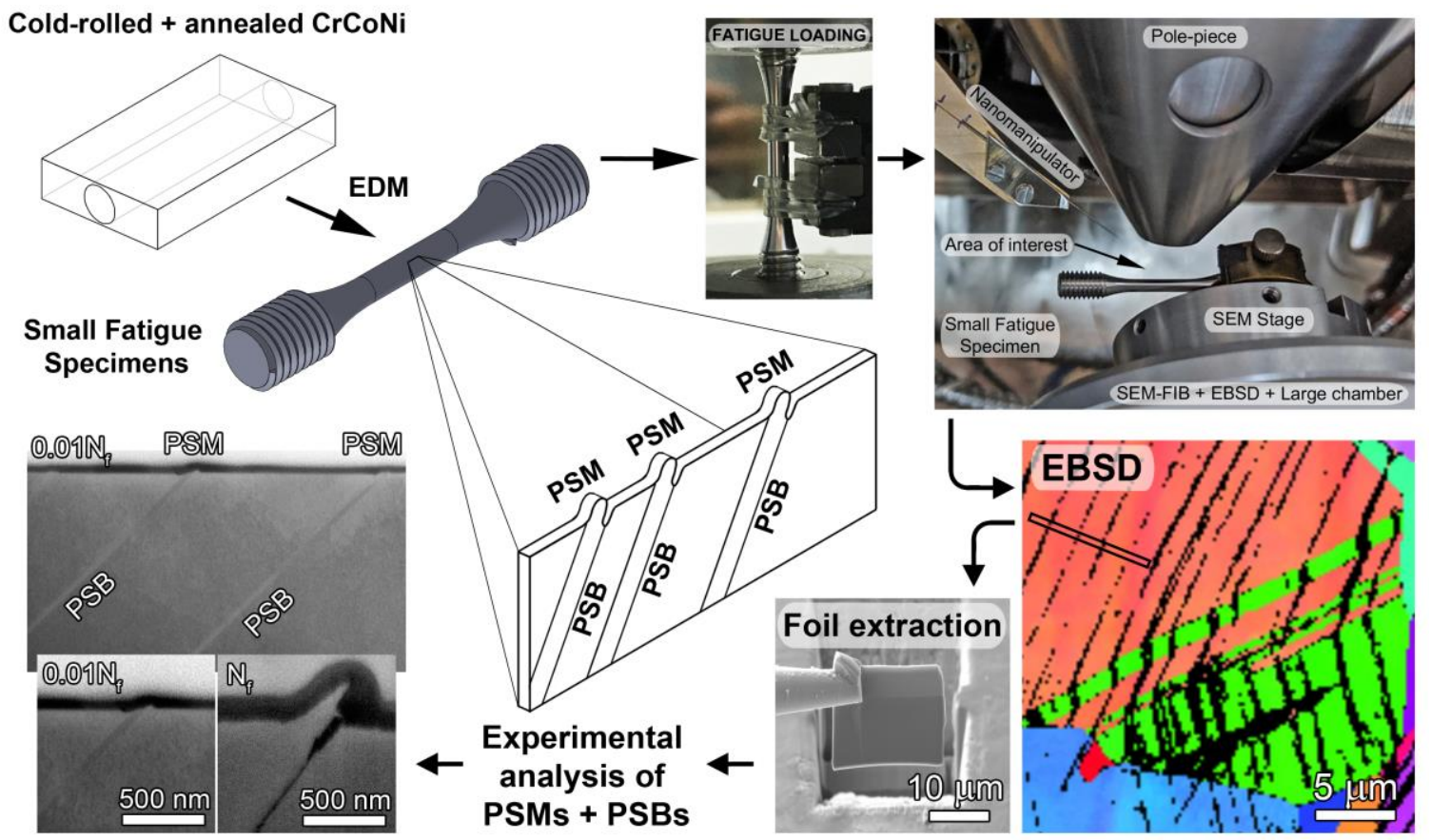

Figure 1. Schematic of framework used to investigate fatigue failure of CrCoNi alloy. Immediately after cyclic loading, specimens are put into the SEM-FIB dual-beam system with EBSD and large chamber. EBSD, ECCI and FIB are used to identify the sites of the interest. Site- and orientation-specific TEM surface lamellae are extracted by FIB. The surface relief and the corresponding underlying microstructure are analyzed by SEM adn STEM-DCI.

\section{References}

[1] Polák and Man, Int. J. Fat. 91 (2) (2016), p. 294-303.

[2] Polák et al., Fat. Fract. Eng. Mat. \& Struct. 40 (7) (2017), p. 1101-1116.

[3] Slone et al., Acta M. 165 (2019), p. 496-507.

[4] The authors acknowledge support from the Thermo Fisher Scientific \& Czechoslovak Microscopy Society fellowship awarded to Milan Heczko. 